\title{
Oxidative Stress in Urinary Tract Infection
}

\author{
Shivendra Dutt ${ }^{1}$, Afreen Arshad Choudhry ${ }^{2}$ \\ ${ }^{1}$ Department of Microbiology, G.C.R.G Institute of Medical Sciences - Lucknow \\ ${ }^{2}$ Department of Biochemistry, MLN Medical College - Allahabad
}

\begin{abstract}
Aerobic respiration and oxidation of bio molecules used as substrates, lead to production of reactive oxygen species (ROS) to combat which we have antioxidant defence mechanisms in our body. Lipid peroxidation is one of the most important expression of oxidative stress induced by ROS. Malonaldehyde (MDA), a product of lipid peroxidation along with antioxidant protein thiols are expressed in plasma. Urinary tract infections (UTIs) may appear in all age groups due to asymptomatic bacteria. The present study aims to determine the relationship of antioxidant levels and oxidative stress in UTI. 60 subjects between the age group 35- 60 years who were diagnosed with Urinary Tract Infection and attended the OPD along with 40 normal healthy subjects (as controls) were included in this study. Midstream urine was sent to microbiology laboratory for diagnosis of UTI. Macroscopic appearance of urine was also studied. MDA were assessed by thiobarbituric acid as a substrate and thiol were estimated by Ellmann's method. Data were analyzed using Statistical Package for the Social Sciences (SPSS 16). In this study we found that plasma MDA level was significantly higher and plasma thiols significantly lower in positive urine cultures compared to negative urine cultures and may indicate the existence of oxidative stress. Since MDA and protein thiols test results can be obtained before the results of cultures taken in urinary tract infection, it may be used as a diagnostic tool and may contribute to the initiation of treatment without waiting for the culture results.
\end{abstract}

Keywords: Urinary tract infection, Oxidative stress

\section{Introduction}

Aerobic respiration and oxidation of bio molecules used as substrates, lead to production of reactive oxygen species (ROS) like hydroxyl radicals, superoxide anions, and hydrogen peroxide to combat which we have Antioxidant defence mechanisms in our body. [1,2] Low levels of ROS are necessary for several biological processes, however increased formation of ROS i.e. Oxidative stress can damage the crucial bio molecules.[3] Lipid peroxidation is one of the most important expression of oxidative stress induced by ROS. Malonaldehyde (MDA), a product of lipid peroxidation along with antioxidant protein thiols are expressed in plasma. Urinary tract infections (UTIs) may appear in all age groups due to asymptomatic bacteria.

Previous researches have not investigated antioxidant enzyme activities in the form of Thiols and MDA levels quantitatively both in diagnosis of UTI and as a response to oxidative stress. The present study therefore aims to determine the relationship of antioxidant levels and oxidative stress in UTI.

\section{Materials and Methods}

Institutional Ethics Committee gave permission to carry out this study. Sixty subjects between the age group 35- 60 years who were diagnosed with Urinary Tract Infection and attended the OPD at Kasturba Hospital along with 40 normal healthy subjects (as controls) consented to participate in this study.

Midstream urine was sent to microbiology laboratory and processed as per standard operating procedure for diagnosis of UTI. Macroscopic appearance of urine was also studied. MDA were assessed by thiobarbituric acid as a substrate and thiol were estimated by Ellmann's method. Data were analyzed using Statistical Package for the Social Sciences (SPSS 16).

\section{Results}

The correlation between semi quantitative colony count in urine and plasma MDA was statistically very significant ( $\mathrm{r}$ value $=0.77, \mathrm{p}<0.001$, Table 1 ) whereas the same was found to be weakly significant in the control group ( $\mathrm{r}$ value= $0.45, \mathrm{p}<0.001$,Table 2). Also the negative correlation between semi quantitative colony count in urine and plasma Thiols was very significant $(\mathrm{r}$ value $=-0.70, \mathrm{p}<0.001$,Table 1 ) whereas the same was found to be weakly significant in the control group $(\mathrm{r}$ value $=0.40, \mathrm{p}<0.001$, Table 2 )

\section{Tables}

Table 1: Correlation of semi quantitative colony count in urine with plasma MDA plasma thiols in patient group

\begin{tabular}{|c|c|c|}
\hline Parameters (in patient group) & $\begin{array}{c}\text { r VALUE (Pearson's } \\
\text { Correlation coefficient) }\end{array}$ & $\begin{array}{c}\mathrm{p} \\
\text { Value }\end{array}$ \\
\hline $\begin{array}{c}\text { colony count in urine and } \\
\text { plasma MDA }\end{array}$ & 0.77 & $<0.001$ \\
\hline $\begin{array}{c}\text { colony count in urine and } \\
\text { plasma Thiols }\end{array}$ & -0.70 & $<0.001$ \\
\hline
\end{tabular}

Table 2: Correlation of semi quantitative colony count in urine with plasma MDA plasma thiols in control group

\begin{tabular}{|c|c|c|}
\hline Parameters (in control group) & $\begin{array}{c}\text { r Value (Pearson's } \\
\text { Correlation coefficient) }\end{array}$ & $\begin{array}{c}\mathrm{p} \\
\text { Value }\end{array}$ \\
\hline $\begin{array}{c}\text { colony count in urine and } \\
\text { plasma MDA }\end{array}$ & 0.45 & $<0.001$ \\
\hline $\begin{array}{c}\text { Urine colony count in urine } \\
\text { and plasma Thiols }\end{array}$ & 0.40 & $<0.001$ \\
\hline
\end{tabular}

\section{Discussion}

Oxidative stress which is an important feature of many diseases is characterized with Increased formation of ROS or decreased antioxidant defence. A variety of oxidation products are found in urine and thought to mirror local and systemic oxidative stress . In the present study we thought that UTI may cause an oxidative stress, and also the 


\section{International Journal of Science and Research (IJSR) \\ ISSN (Online): 2319-7064 \\ Index Copernicus Value (2015): 78.96 | Impact Factor (2015): 6.391}

antioxidant enzymes measured quantitatively were depleted in response to oxidative stress. Kirschbaum reported that total antioxidant enzyme activity was lower in patients with acute renal disease compared to those of control urine specimen [4]. In our study the results show that UTI may cause oxidative stress by consuming urinary antioxidant enzymes and it is possible to say that urinary antioxidant enzymes are not enough to prevent the oxidative stress in UTI. According to our study, overproduction of free radicals generated during infection may lead to the low levels of antioxidant enzymes. Since the start of antibiotic therapy according to the culture results may take time, antioxidant agents such as vitamin $\mathrm{C}$ may be given promptly in addition to the antibiotic therapy[5].

MDA is an indicator of lipid peroxidation in cell and tissues and has been used as a marker of oxidative stress. Plasma MDA is found in increased quantities in some diseases such as thalassemia, renal failure, and pancreatic disease. Antioxidants like protein thiols are entities whose presence in relatively high concentration significantly inhibits the rate of oxidation of bio molecules. By acting as potent electron donors, antioxidants donate hydrogen atoms to pair up with unpaired electrons in free radicals thereby converting reactive free radicals into inactive form. The determination of oxidative stress and antioxidants in plasma can indicate disease progression and prognosis. In this study we found that plasma MDA level was significantly higher and plasma thiols significantly lower in positive urine cultures compared to negative urine cultures and may indicate the existence of oxidative stress. Since MDA and protein thiols test results can be obtained before the results of cultures taken in urinary tract infection, it may be used as a diagnostic tool and may contribute to the initiation of treatment without waiting for the culture results.

\section{Conclusion}

UTI may cause oxidative stress and increase lipid peroxidation level leading to a decrease of antioxidant enzymes. We believe that patients with UTI may benefit from antioxidant treatments in addition to antibacterial treatment.

\section{References}

[1] Ergul Belge Kurutas, Pınar Ciragil, Mustafa Gul, Metin Kilinc. The Effects of Oxidative Stress in Urinary Tract Infection. Mediators Inflamm. 2005 Aug 31; 2005(4): 242-244.

[2] Wael M. El-Deeb, Sébastien Buczinski. The diagnostic and prognostic importance of oxidative stress biomarkers and acute phase proteins in Urinary Tract Infection (UTI) in camels. PeerJ. 2015; 3: e1363.

[3] Kang HK, Kim DK, Lee BH, et al. Urinary N-acetylbeta-D-glucosaminidase and malondialdehyde as a markers of renal damage in burned patients. $J$ Korean Med Sci. 2001;16(5):598-602.

[4] Kirschbaum B. Total urine antioxidant capacity. Clin Chim Acta. 2001;305(1-2):167-173.

[5] Shivendra Dutt , Nagalakshmi N , Shobha K. L. Small colony variant of Escherichia coli isolated from a recurrent urinary tract infection- a case report. Intl $J$ of Health. 2 (2) ,2014, 61-62. 